\title{
Geschlechterrollen im römischen Erbrecht im Spiegel des zeitgenössischen Gerechtigkeits- verständnisses und am Beispiel der lex Voconia
}

\author{
Elena Köstner
}

Jede Zeit hat ihre sozialen Normen und Wertvorstellungen und das spiegelt sich wieder in der zeitgenössischen Gesetzgebung und im Verständnis von Gerechtigkeit. Neue Gesetze können gewöhnlich als Reaktion auf bereits erfolgte Entwicklungen gesehen werden. Der Gesetzgeber reagiert damit auf soziale, ökonomische und politische Veränderungen. Das trifft auch auf das römische Erbrecht zu. Der Fokus meiner Untersuchung liegt dabei auf dem römischen Konzept von Weiblichkeit, das in Gesetzen zum römischen Erbrecht - im Besonderen im Fall der lex Voconia - ersichtlich wird sowie auf seiner Reflexion im zeitgenössischen Gerechtigkeitsverständnis.

Im römischen Kulturkreis existierte eine ausgeprägte Ambivalenz der Genderverhältnisse: Die römische Gesellschaft war patriarchalisch organisiert, was sich einerseits in ihren Gesetzen widerspiegelt und darin, dass Frauen von bestimmten Bereichen der gesellschaftlichen Partizipation und Interaktion ausgeschlossen blieben (sehe auch der Beitrag von Pavon). So durften Frauen nicht wählen oder wählbar sein, keine Ämter in der res publica bekleiden oder in den Streitkräften tätig sei. ${ }^{1}$ Jedoch haben Frauen am Wirtschafts- und

1 Vgl. Dig. 50.17.2pr.-1; N. Benke, ,Gender and the Roman law of obligations', in Th.A.J. McGinn (Hrsg.), Obligations in Roman Law, Past, Present, and Future, Papers and Monographs of the American Academy in Rome 33 (Ann Arbor 2012), 215-246: 220; J.F. Gardner, ,Gender-role assumptions in Roman law', Echos du monde classique 39 (1995), 377-40o: 377-378; N. Benke und V.T. Halbwachs, Rezension zu G. Rizzelli, Le donne nell' esperienza giuridica di Roma antica. Il controllo dei comportamenti sessuali. Una raccolta di testi, Zeitschrift der SavignyStiftung für Rechtsgeschichte 119 (2002), 472; C.W. van Galen, Women and Citizenship in the Late Roman Republic and the Early Empire (Nijmegen 2016), 51; Barbara Levick (,Women and law', in: S.L. James und S. Dillon (Hrsg.), A Companion to Women in the Ancient World (Malden, Oxford, Chichester 2012), 96-106: 98) findet folgende Erklärung für den Ausschluss von Frauen von der Bekleidung öffentlicher Ämter: Als ursprünglich militärisch orientierte 
Rechtsleben partizipiert. Nach Nikolaus Benke wurden rechtliche Artikulationen erzeugt, die Frauen gegenüber Männern ausdrücklich unter Bezugnahme auf die Geschlechtszugehörigkeit unterschieden. ${ }^{2}$ Als sui iuris war die römische Frau von manus oder patria potestas befreit und nur noch der tutela mulierum unterworfen, wobei dieses Kontrollinstrument nicht zu allen Zeiten regulierend eingriff. Sie war vermögens- und verpflichtungsfähig, d.h. sie konnte Eigentum besitzen und verkaufen, Verhandlungen tätigen und Verträge schließen. Diese Ambivalenz findet sich auch im römischen Erbrecht wieder. Seit der römischen Frühzeit und den Zwölf Tafeln hatten Frauen umfassende Rechte im Rahmen der gesetzlichen Erbfolge. ${ }^{3}$ Die römische Erbrechtsordnung basierte auf der agnatischen Familienstruktur: Nach dem ius civile waren nur Agnaten (in erster Linie die sui heredes) erbberechtigt, falls kein Testament vorlag. Unter den sui heredes waren Söhne und Töchter des Erblassers gleichberechtigt. Doch kam es in der Folgezeit zu Einschränkungen, die u.a. mit dem Geschlecht der Erbin bzw. des Erben begründet wurden, wobei die Motive dafür vielmehr in sozialen und politischen Veränderungen zu suchen sind: "While the agnate system of succession lost considerable ground to the cognate, the structure of the Roman family and of Roman property-holding remained essentially patriarchal throughout the classical period." ${ }^{4}$ Die Gesetze, die sich auf das testamentarische Erbrecht bezogen und die uns zumindest in Teilen überliefert sind, sind die lex Furia, lex Voconia und lex Falcidia. Ihr Anliegen war es, testamentarische Legate zu begrenzen. Der Interpretation des Gaius folgend sollte auf diese Weise das Familienvermögen konserviert und den Erben ein Minimum an Vermögen zugesichert werden. ${ }^{5}$ Von diesen

Gemeinschaft gestand Rom nur denjenigen politische Macht und Ämter zu, die für die Gemeinschaft gekämpft hatten oder kämpfen konnten und das waren Männer.

2 Vgl. Dig. 1.5.5pr., 1.5.9; Benke und Halbwachs 2002, a.a. O. (Anm. 1), 470; im Gegensatz dazu vgl. Detlef Liebs (,Römische Gerechtigkeit durch fairen Prozess. Juristen mit Autorität und allgemeingültige Maßstäbe', in: I. de Gennaro (Hrsg.), Value, Sources and Readings on a Key Concept of the Globalized World (Leiden, Boston 2012), 57-82: 78-79), der Rom eine Gleichstellung von Männern und Frauen vor dem Gesetz attestiert, da agnatisch verwandte Frauen im Erbfall ebenso viel erbten wie Männer. Liebs Ansatz greift zu kurz, da er sich nur auf die gesetzliche, nicht aber auch auf die testamentarische Erbfolge fokussiert.

3 Vgl. XII Tab. 5.3-7.

4 J.F. Gardner, Women in Roman Law and Society (London, Sydney 1986), 163.

5 Vgl. Gai. Inst. 2.224-227; Cic. leg. 2.48-53; U. Wesel, ,Über den Zusammenhang der lex Furia, Voconia und Falcidia', Zeitschrift der Savigny-Stiftung für Rechtsgeschichte 81 (1964), 308-316; J.F. Gardner, ,Nearest and dearest: liability to inheritance tax in Roman families', in: S. Dixon (Hrsg.), Childhood, Class and Kin in the Roman World (London, New York 2001), 205-220: 213; J.F. Gardner, Family and Familia in Roman Law and Life (Oxford, New York 1998), Gardner 1998, a. a. O., 15-46; Van Galen 2016, a.a. O. (Anm. 1), 116-117, 177. 
Gesetzen weist lediglich die lex Voconia eine weitere Klausel auf, nämlich ein Erbeinsetzungsverbot von Frauen, das jedoch nur Erblasser der 1. Censusklasse betraf. $^{6}$

Zur Untersuchung von Genderrollen und zeitgenössischem Gerechtigkeitsverständnis möchte ich das Augenmerk auf die Nutzung von exempla in der historiographischen und juristischen Narration lenken, da diese in diesem Kontext eine nicht zu unterschätzende Rolle einnehmen. Es ist eine weit verbreitete und kulturübergreifende Tradition, mythische oder historische Figuren, Handlungen oder Ereignisse als exempla - Vorbilder bzw. Rollenbilder zu nutzen, da ihnen ein spezifischer kultureller Habitus innewohnt, der helfen kann, gesellschaftliche Unterschiede und Umbrüche erklärbar zu machen. ${ }^{7}$ Ein exemplum oder Vorbild ist also etwas oder jemand, das oder der kopiert werden kann. Es verkörpert ein Ideal mit normativem Charakter, das eingesetzt werden kann, um soziale Kohäsion herzustellen oder zu vertiefen. ${ }^{8}$ Gleichzeitig werden von einem Vorbild tatsächlich nur ein oder zwei Verhaltensweisen bzw.

6 Vgl. Paul. Sent. 4.8.20; Gai. Inst. 2.274; Gardner 1986, a.a. O. (Anm. 4), 170-179; R. Vigneron und J.-F. Gerkens, ,The emancipation of women in Ancient Rome', Revue Internationale des droits de l'antiquité 47 (2000), 107-121; J.A.J.M. van der Meer, The Lex Voconia: Made for Men. Mulier heres institui non potest (Eijsden 1996), 5-13, 23-43; A. Weishaupt, Die lex Voconia, Forschungen zum Römischen Recht 45 (Köln, Weimar, Wien 1999), 40-54; 107-116; N. Benke, Rezension zu A. Weishaupt, Die lex Voconia, Zeitschrift der Savigny-Stiftung für Rechtsgeschichte 119 (2002), 488-510; A. McClintock, ,The lex Voconia and Cornelia's jewels', Revue internationale des droits de l'antiquité 6o (2013), 183-200: 186-188; B. Hopwood, ,Livia and the lex Voconia', in E. Herring und K. Lomas (Hrsg.), Gender Identities in Italy in the First Millenium BC (Oxford 2009), 143-148; J.P. Hallett, Fathers and Daughters in Roman Society, Women and the Elite Family (Princeton 1984), 92-95; E. Baltrusch, Regimen morum. Die Reglementierung des Privatlebens der Senatoren und Ritter in der römischen Republik und frühen Kaiserzeit, Vestiga 41 (München 1989), 75-77; K. Verboven, The Economy of Friends. Economic Aspects of Amicitia and Patronage in the Late Republic, Collection Latomus 269 (Brüssel 2002) 221; S.B. Pomeroy, ,The relationship of the married woman to her blood relatives in Rome', Ancient Society 7 (1976), 215-227: 222; R. Vigneron, ,L' antiféministe loi Voconia et les "Schleichwege des Lebens"', Labeo 29 (1983), 140-153; J. Pölönen, ,Lex Voconia and conflicting ideologies of succession, privileging agnatic obligation over cognatic family feeling', Arctos 33 (1999), 111-131; S. Dixon, ,Breaking the law to do the right thing. The gradual erosion of the Voconian Law in Ancient Rome', Adelaide Law Review 9 (1985), 519-534; J.K. Evans, War, Women and Children in Ancient Rome (London, New York 1991), 75-76; A.J.B. Sirks, ,Sacra, succession and the lex Voconia', Latomus 53 (1994), 273-296; S. Hähnchen, ,Ratio Voconiana. Gedanken zur erbrechtlichen Benachteiligung', in: J.D. Harke (Hrsg.), Facetten des römischen Erbrechts. Studien zur Geschichte und Dogmatik des Privatrechts (Berlin, Heidelberg 2012), 35-54: 39-41, $46-48$.

7 Vgl. S. Bell, ,Role model in the Roman world', in: S. Bell und I.L. Hansen (Hrsg.), Role Models in the Roman World. Identity and Assimilation (Ann Arbor 2008), 1-40: 2.

8 Vgl. Bell 2008, a. a. O. (Anm. 7), 4; Liv. 1.10-11. 
Charakteristika situationsspezifisch zur Nachahmung ausgewählt, weshalb sie eine stereotypisierte Konnotation erhalten. Werden exempla in eine Narration integriert, bieten diese moralische Orientierung, die nicht auf die Vergangenheit beschränkt sein muss, denn es handelt sich vielmehr um eine Interaktion zwischen einem exemplum der Vergangenheit und den Rezipienten der Gegenwart. In diesen Dialog möchte ich die Frage nach Geschlechterrollen und dem Konzept von Weiblichkeit im römischen Erbrecht verorten sowie das zeitspezifische Gerechtigkeitsverständnis.

In das Spannungsverhältnis von Recht und Gesetz wirkt das Konzept der Gerechtigkeit ein, das sich auf das zwischenmenschliche Verhalten bzw. das Verhältnis zwischen Menschen und Normen bzw. Normsystemen bezieht. „Gerechtigkeit impliziert einen Maßstab, der im Verhältnis der Menschen zueinander und ihrer Ordnung auf ein angemessenes Verhältnis von Geben und Nehmen, von Fordern und Verweigern abzielt..9 Gerechtigkeit kann also auch als reziprokes Prinzip verstanden werden, das der Einhaltung von Recht dient. Sowohl in der platonischen als auch in der aristotelischen Ideenlehre wird Gerechtigkeit ( $\delta w \alpha 10 \sigma \dot{v} \eta \eta)$ als oberste Tugend ( $\alpha \rho \varepsilon \tau \eta \dot{ })$ verstanden, die einerseits als Richtlinie für das Verhalten des Individuums im Sozialen fungiert, andererseits innerhalb des idealen Staatsgefüge. ${ }^{10}$ Gerechtigkeit wird darin ersichtlich, dass jede und jeder die ihr oder ihm zugewiesenen Aufgaben erfüllt. Die grundlegenden Aspekte von Gerechtigkeit wie Gleichheit, Gegenseitigkeit, Leistungsausgleich und Entgeltlichkeit wurden erstmals im römischen Recht realisiert, genauso wie der Anspruch auf Gerechtigkeit per se. Nach Ulpian sei Gerechtigkeit der beständige und unveränderliche Wille, einem jeden das Seinige zukommen zu lassen:

Vgl. W. Brugger, ,Gesetz, Recht, Gerechtigkeit', Humanistische Bildung 13 (1989), 65-85: 66-67, 70-71; U. Kornblum, ,Bemerkungen zum Thema “Recht und Gerechtigkeit”', Humanistische Bildung 13 (1989), 7-26: 7-8.

10 Vgl. Plat. Pol. 4.433a, 453a-d; Aristot. Eth. Nic. 5·3.1129b, 5.10.1137b17-18; H.W. Arndt, ,Philosophische Aspekte des Begriffs Gerechtigkeit', Mannheimer Berichte aus Forschung und Lehre 21 (1981), 591-598: 591; G. Santas, ,Justice and gender in the laws and the Republic', in: S. Scolnicov und L. Brisson (Hrsg.), Plato's Laws. From Theory to Practice. Proceedings of the 6th Symposium Platonicum (Sankt Augustin 2003), 237-242: 237-238; J. Barnes, ,Justice writ large', in: R. Kamtekar (Hrsg.), Virtue and Happiness: Essays in Honour of Julia Annas (Oxford 2012), 31-50: 31-37. Der Terminus „der Gerechte“ bedeutet dann die strukturelle Zugehörigkeit und Zuordnung zu einem gesellschaftlichen Ganzen (vgl. Plat. Pol. 4.435e436a). Die drei Stände in Platons Idealstaat K $\alpha \lambda i \pi 0 \lambda ı$ entsprechen den drei Seelenteilen (vgl. Plat. Pol. $4.438 \mathrm{~d}-441 \mathrm{c}, 4.443 \mathrm{c}-445 \mathrm{e}, 4.58 \mathrm{oe}-581 \mathrm{a}-\mathrm{e})$. 
iustitia est constans et perpetua voluntas ius suum cuique tribuendi.

Gerechtigkeit ist der unwandelbare und dauerhafte Wille, jedem sein Recht zu gewähren."1

In diesem Verständnis erscheint Gerechtigkeit als Tugend, aber nicht als abstraktes Prinzip. ${ }^{12}$ Als eine kultur- und zeitübergreifende Prämisse kann eine enge Verbindung zwischen Recht und Gerechtigkeit attestiert werden, wobei Gerechtigkeit objektiv als "die inhaltliche Richtigkeit des Rechts" und subjektiv als "die Rechtschaffenheit einer Person“ verstanden wird. ${ }^{13}$ Gerade die objektive Komponente des Gerechtigkeitsbegriffs kann als „ein Grundbegriff menschlichen Verlangens" verstanden werden, d.h. der Mensch sehne sich danach, würde es aber auch gleichzeitig einfordern. ${ }^{14}$ Durch diese ethische Konnotation wirkt Gerechtigkeit als Konnektiv von Recht und Gesetz, als theoriebezogener Leitgedanke und schafft gleichzeitig eine Verbindung zu Gesellschaft und sozialer Realität. Aufgrund dieser Prämissen stellen sich mir folgende Fragen hinsichtlich der Rolle von Frauen im römischen Erbrecht und im Besonderen im Fall der lex Voconia: (1) Unter welchen Bedingungen wird Gerechtigkeit herausgefordert? (2) Ist die lex Voconia ungerecht? (3) Gibt es im römischen Erbrecht im Bereich der Gesetzgebung zu Testamenten eine genderspezifische iustitia?

2

\section{Intentionen einer Erbschaft und familiäre Strategien zur testamentarischen Vererbung}

In seiner philosophischen Abhandlung De finibus bonorum et malorum spricht Cicero mit zwei Freunden des Brutus u.a. auch über das Abfassen von Testamenten:

quoniamque illa vox inhumana et scelerata ducitur eorum, qui negant se recusare quo minus ipsis mortuis terrarum omnium deflagratio consequatur $[\ldots]$, certe verum est etiam iis, qui aliquando futuri sint, esse prop-

11 Dig. 1.1.10 (Corpus Iuris Civilis, Text und Übersetzung, Digesten 1-10, übers. und hrsg. von O. Behrends, R. Knütel, B. Kupisch, H.H. Seiler (Heidelberg 1995)); vgl. Cic. Inv. 2.53.16o; Rep. 3.11.18; Leg. 1.6.19; Off. 1.5.15.

12 Vgl. Cic. Leg. 1.45; W. Waldstein, Was ist Gerechtigkeit? (Zu Ulpians Definition, Digesten 1.1.1opr.)‘, Wiener Humanisitische Blätter 21 (1979), 1-16: 10.

13 O. Höffe, Gerechtigkeit. Eine philosophische Einführung (München 2001), 9.

14 Höffe 2001, a.a. O. (Anm. 13), 9. 
ter ipsos consulendum. ex hac animorum affectione testamenta commendationesque morientium natae sunt.

So hält man denn auch jene Äußerung für unmenschlich und verbrecherisch, in der einer erklärt, ,es sei ihm durchaus recht, wenn nach seinem Tode die ganze Welt in Flammen aufginge. [...] Da außerdem niemand in der völligen Einsamkeit sein Leben zu führen wünscht, nicht einmal in einer unendlichen Fülle an Lust, so ergibt sich leicht, daß wir zur Verbindung und Geselligkeit mit den Menschen und zu einer naturgemäßen Vergesellschaftung geboren sind. ${ }^{15}$

Die Verantwortung des Erblassers für die Hinterbliebenen kollidierte also mit seinem Recht aus den Zwölf Tafeln, frei und widerrufbar über die Verteilung seines Vermögens und die Erbfolge zu entscheiden, wobei Landbesitz eine nicht unwesentliche Rolle spielte, galt dieser doch nicht nur als vorherrschende Vermögensform und bevorzugtes Investment, sondern auch als begehrtes Erbgut. Dabei war es wichtig, diesen Landbesitz über Generationen für die Familie zu bewahren, denn sozialer Status und Prestige waren nicht vollumfänglich vererbbar, sondern u.a. an Eigentum geknüpft. ${ }^{16}$ In diesem Zusammenhang kann familia als ein Syndikat mit drei wesentlichen Zielen verstanden werden: Zum einen dem Erhalt des Familienkults, zum anderen zur Vergrößerung des Wohlstands und des Weiteren zum Erwerb von Ansehen. ${ }^{17}$ Dieses Bestreben sollte erreicht werden durch die patria potestas des pater familias sowie durch die Separierung von Vermögen, wenn zwei Familien durch Heirat miteinander verbunden wurden. ${ }^{18}$ Die Rolle der römischen Frau in diesem familia-Konzept

15 Cic. Fin. 3.64-65 (M. Tullius Cicero, De finibus bonorum et malorum. Über die Ziele menschlichen Handelns, hrsg., übers. und komm. von O. Gigon und L. Straume-Zimmermann, Sammlung Tusculum, (München, Zürich 1988)); vgl. XII Tab. 5,3.

16 Vgl. R.P. Saller, Roman heirship strategies in principle and in practice', in: D.I. Kertzer und R.P. Saller (Hrsg.), The Family in Italy from Antiquity to the Past (New Haven, London 1991), 26-46: 26-27; Levick 2012, a.a. O. (Anm. 1), 10o; Wesel 1964, a.a. O. (Anm. 5), 308ff.; Van Galen 2016, a. a. O. (Anm. 1), 84-94; Gai. Inst. 2.224, 3.1, 3.3; XII Tab. 5.3; Paul. Sent. 4.8.20; Iust. inst. 3.2.3a; Cod. Iust. 6.58.14; Dig. 50.16.195.2; 50.16.195.4-5.

17 Vgl. N. Benke, Why should the law protect Roman women? Some remarks on the Senatus Consultum Velleianum (ca. 5o A.D.)', in: K.E. Børresen, S. Cabibbo and E. Specht (Hrsg.), Gender and Religion Genre et religion (Rom 2001), 41-56: 43-44; Van Galen 2016, a.a.O. (Anm. 1), 6o-62.

18 In der familia communi iure machte der Tod des pater familias alle Agnaten zu sui iuris. Agnation war v. a. für die gesetzliche Erbfolge von Bedeutung, da an erster Stelle die hauseigenen, gewaltunterworfenen Personen standen, also die sui iuris. „[...] nach diesem Prinzip [werden] Männer und Frauen gleich behandelt". (E. Höbenreich und G. Rizzelli, 
bestand einerseits darin, Kinder zu bekommen und andererseits den guten Ruf der Familie zu bewahren. Letzteres galt sowohl für ihre Handlungen innerhalb als auch außerhalb der domus. Römische Frauen waren zwar freie Bürgerinnen, aber ihre Rechte waren eingeschränkt. Solange Frauen unter der patria potestas ihres Vaters oder der manus ihres Ehemannes standen, waren sie nicht rechtsfähig, unfähig eigenes Vermögen zu besitzen, denn ihr ganzer Besitz gehörte dem pater familias bzw. dem Ehemann. ${ }^{19}$

\section{Das Erbeinsetzungsverbot der lex Voconia und die Rolle der Frau im römischen Erbrecht}

Die lex Voconia wurde 169 v. Chr. von Q. Voconius Saxa als Plebiszit eingebracht und von M. Porcius Cato unterstützt. ${ }^{20}$ Neben einer Beschränkung testamentarischer Legate beinhaltete dieses Gesetz noch eine weitere Klausel: Frauen durften von Bürgern der 1. Censusklasse nicht mehr als Erbinnen eingesetzt werden..$^{21}$

Scylla. Fragmente einer juristischen Geschichte der Frau im antiken Rom (Wien, Köln, Weimar 2003), 14; vgl. Levick 2012, a. a. O. (Anm. 1), 99; S. Dixon, The Roman Mother (London 1988), 44; Gai. Inst. 1.55). Zur potestas des pater familias vgl. B.D. Shaw, ,Raising and killing children: two Roman myths', Mnemosyne 54 (2001), 31-77; Van Galen 2016, a.a. O. (Anm. 1), 68-71.

19 Es existierte die Möglichkeit, ein peculium zu erhalten, also eine Art Sondervermögen, das der pater familias an eine gewaltunterworfene Person übergab und das diese dann selbständig verwalten konnte. Rechtlich blieb der pater familias Eigentümer und verfügungsberechtigt (vgl. Dig. 34.4.31.3; Gardner 1986, a.a.O. (Anm. 4), 9; Levick 2012, a.a.O. (Anm. 1), 99; Pomeroy 1976, a. a. O. (Anm. 6), 215, 222; Plut. Rom. 22,3).

20 Vgl. Liv. Per. 41; Cic. Cato 5,14; Gell. 7.13.3; zu dem Volkstribunen Voconius vgl. Cic. Balb. 8.21; Ps.-Asconius, Cic. Verr. 2.1.41.106; zu Cato als Unterstützer der lex Voconia vgl. Gell. 6.13.3, 17.6.1. Zur Einschätzung der Quellen zur lex Voconia vgl. Weishaupt 1999, a. a. O. (Anm. 6), 1-34.

21 Der freie Gestaltungswille des Erblassers aus den Zwölf Tafeln wurde hinsichtlich der Legate durch die lex Furia testamentaria, die lex Voconia und die lex Falcidia eingeschränkt: vgl. Gai. Inst. 2.224-227, 3,14; XII Tab. 5.3; Dig. 35.2.1pr, 35.2.73.5; Inst. Iust 2.22; Theoph. Inst. paraphrasis 2.22; Cic. Verr. 2.1.43.110; Weishaupt 1999, a.a.O. (Anm. 6), 6, 73-101; 122-124; Hähnchen 2012, a.a. O. (Anm. 6), 38-40; Baltrusch 1989, a.a. O. (Anm. 6), 70-77; Wesel 1964, a.a.O. (Anm. 5), 308-314; Van der Meer 1996, a.a. O. (Anm. 6), 14-25; Vigneron und Gerkens 2000, a. a. O. (Anm. 6), 107-121; P. Stein, ,Lex Falcidia', Athenaeum 65 (1987), 453-457: 453. Eine dritte Klausel der lex Voconia wird bei Paul. Sent. 4.8.20 genannt. Sie beinhaltet, dass Frauen nicht nur als testamentarische Erbinnen eingeschränkt würden, sondern auch in der gesetzlichen Erbfolge. Auch die declamationes minores des Quintilian (264) nennen eine dritte Klausel, die besagen soll, dass einer Frau generell nicht mehr als die Hälfte des Vermögens vermacht werden dürfe. Zu einer potentiellen dritten 
Item mulier, quae ab eo, qui centum milia aeris census est, per legem Voconiam heres institui non potest, tamen fideicommisso relictam sibi hereditatem capere potest.

Ferner kann eine Frau zwar von jemandem, dessen Vermögen auf 100 ooo As eingeschätzt worden ist, aufgrund des Voconischen Gesetzes nicht zur Erbin eingesetzt werden, doch kann sie eine Erbschaft, die ihr durch ein Fideikommiss hinterlassen worden ist, erwerben. ${ }^{22}$

Die Forschung geht mehrheitlich davon aus, dass einerseits jede Frau dem Einsetzungsverbot unterlag, andererseits nur Testatoren der 1. Censusklasse. ${ }^{23}$ War ein Erblasser nicht zensiert, so kam die lex Voconia auch nicht zur Anwendung, selbst wenn ihn sein Vermögen eindeutig für die 1. Censusklasse qualifizierte. ${ }^{24}$ Mit der lex Voconia wurde das Intestaterbrecht der Frau nicht angetastet; eine Beschränkung von Frauen in diesem Bereich wäre jedoch weitaus tiefgreifender gewesen und hätte sie von einer bedeutsamen Vermögensquelle abgeschnitten. ${ }^{25}$

Vor dem Jahr 169 v. Chr. hatte die Senatorenschaft anscheinend keine Bedenken gehabt, Frauen zu Erbinnen zu bestimmen. ${ }^{26}$ Es stellt sich die Frage, was sich de facto oder auch nur im Empfinden der Senatoren geändert hatte, dass

Klausel der lex Voconia vgl. u. a. Van der Meer 1996, a. a. O. (Anm. 6), 23-43; Weishaupt 1999, a. a. O. (Anm. 6), 107-116; Benke 2002, a. a. O. (Anm. 6), 494-495.

22 Gai. Inst. 2.274 (Gaius, Institutiones, hrsg., übers. und komm. von U. Manthe, Texte zur Forschung 81, (Darmstadt 2010)).

23 Vgl. Weishaupt 1999, a.a.O. (Anm. 6), 40-54; Van der Meer 1996, a.a.O. (Anm. 6), 5-13; McClintock 2013, a. a. O. (Anm. 6), 186-188; Hopwood 2009, a. a. O. (Anm. 6), 142 ff.; Gardner 1986, a. a. O. (Anm. 4), 170-179; J. Le Gall, Un critère de différenciation sociales. La situation de la femme. Recherches sur les structures sociales dans l'antiquité classique (Paris 1970), 176177; Hallett 1984, a. a. O. (Anm. 6), 92-95; Hähnchen 2012, a.a. O. (Anm. 6), 39-41; Baltrusch 1989, a.a. O. (Anm. 6), 75-77; Van Galen 2016, a. a. O. (Anm. 1), 177-178; 184; 187.

24 Vgl. Cic. Verr. 2.1.43.104-114. Die Wertgrenze der 1. Censusklasse ist unterschiedlich mit 100.000 Assen (Gai. Inst. 2.274), 125.000 Assen (Gell. 7.13) und 100.00o Sesterzen (Ps.Asconius, Cic. Verr. 2.1.43.104; Cass. Dio 56.10.2) angegeben (vgl. T.P. Wiseman, ,The census in the first century B.C., Journal of Roman Studies 59 (1969), 59-75).

25 Vgl. Weishaupt 1999, a. a. O. (Anm. 6), 54-62. Zur Ahndung von Verstößen gegen die lex Voconia vgl. Weishaupt 1999, a.a. O. (Anm. 6), 116-128; Benke 2002, a. a.O. (Anm. 6), 496; Van der Meer 1996, a.a.O. (Anm. 6), 7-11; Cic. Verr. 2.1.40-44; Plin. Paneg. 42.1. Zu ihrer Geltungsdauer bis ins 2. Jahrhundert n. Chr. vgl. Gell. 20.1.23; Gai. Inst. 2.268, 2.274, 2.284. Für ein Ende der Geltungsdauer der lex Voconia bereits während der späten Republik vgl. Van der Meer 1996, a. a. O. (Anm. 6), 115; Suet. Aug. 27.5; Cass. Dio 52.42.1; Weishaupt 1999, a.a.O. (Anm. 6), 47-51; Wiseman 1969, a.a.O. (Anm. 24), 59-75.

Vgl. Pomeroy 1976, a. a. O. (Anm. 6), 222. 
dieses Gesetz mit dem Erbeinsetzungsverbot von Frauen erlassen wurde. ${ }^{27}$ Wenn es das Ziel der lex Voconia gewesen sein soll, Frauen der Nobilität ihres Vermögens zu berauben, um ihre angebliche superbia und luxuria zu unterbinden, dann war diese Maßnahme nicht zielführend. Denn es gab ausreichend Möglichkeiten, die lex Voconia zu umgehen und Frauen doch noch mit Vermögen zu bedenken. Eine besonders einfallsreiche Strategie verfolgte Aemilia mit ihrem Testament: Als reiche Witwe setzte sie Scipio Aemilianus als Erben ein, hinterließ aber nichts ihrer biologischen Tochter Cornelia, die Mutter der Gracchen und die Ehefrau des Scipio Nasica. Nach dem Tod der Aemilia 162 v. Chr. erklärte Scipio Aemilianus, er würde die Abschlussraten der Mitgiften an seine Adoptivtanten zahlen und so erhielt auch Cornelia ihren Anteil am Vermögen ihrer Mutter. ${ }^{28}$ Auf diese Weise konnte Aemilia ihre Tochter über Umwege letztwillentlich bedenken und die lex Voconia umgehen.

In seinem 1983 erschienenen Aufsatz listet Roger Vigneron diese „Schleichwege des Lebens", wie er es nennt, auf: ein vorsätzliches Umgehen des census, der Verzicht auf ein Testament, das Einrichten eines fideicommissum, das Nutzen eines legatum ususfructus oder legatum partitionis sowie die Entscheidung eines Prätors. ${ }^{29}$ Eine weitere Möglichkeit, die lex Voconia zu umgehen und das Vermögen von Frauen zu vergrößern, bestand darin, Töchtern eine opulente Mitgift auszuhändigen. Die Regularien zur dos wurden auch nach 169 v. Chr. nicht verändert. ${ }^{30}$ Gründe und Motive, die für das Erlassen der lex Voconia in der Forschung bislang gefunden wurden, sind die Begrenzung der Vermögens-

27 Vgl. Cic. Rep. 3.10.17; Verr. 2.1.43, 110-114; Fin. 2.17.55; Balb. 21; Cato 5.14; Cluent. 7.21; Caec. 4.11; Gell. 7.13, 17.6.1, 20.1.23; Aug. Civ. 3.21; Weishaupt 1999, a.a. O. (Anm. 6), 8-19; 26-28; Van der Meer 1996, a. a. O. (Anm. 6), 73-79; Gardner 1986, a.a. O. (Anm. 4), 173-178; Vigneron 1983, a.a. O. (Anm. 6), 144; Vigneron und Gerkens 2000, a. a. O. (Anm. 6), 107-121. Zum fideicommissum: Cicero hatte ein fideicommissum für Publilia erhalten, doch er heiratete sie und erhielt den Erbteil des fideicommissum durch ihre Mitgift (Plut. Cic. 41.4).

28 Vgl. Polyb. 31.26.1, 31.28.13; A. McClintock, ,Polyb. 31.26-28: la successione di Emilia', Index 33 (2005), 317-336; Hähnchen 2012, a.a. O. (Anm. 6), 46-48.

29 Zu den Tricks, die lex Voconia zu umgehen vgl. Gai. Inst. 2.224; Dig. 35.2.1.7; Cic. Att. 1.18; Verr. 2.1.43.110-114; rep. 3.10; fin. 2.17.55; Caec. 4.11, 4.10; Cluent. 7.21; Vigneron 1983, a.a.O. (Anm. 6), 140-153; Weishaupt 1999, a.a. O. (Anm. 6), 41; 108-115; Verboven 2002, a.a. O. (Anm. 6), 220 ff.; Evans 1991, a. a. O. (Anm. 6), 76-77: „The Voconian law appears, therefore, to have been a dead letter literally from the moment of its inception."

3o Pomeroy 1976, a.a.O. (Anm. 6), 221-222; Van Galen 2016, a.a.O. (Anm. 1), 111-114. Während des 2. Punischen Kriegs waren so viele Männer gestorben oder in Gefangenschaft geraten, so dass das tributum nicht die Ausgaben des Staates decken konnte (Liv. 23.48.89; Van Galen 2016, a.a. O. (Anm. 1), 20; S. Northwood, ,Census and tributum', in: L. de Ligt und S. Northwood (Hrsg.), People, Land, and Politics. Demographic Developments and the Transformation of Roman Italy 30о BC-AD 14 (Leiden 2008), 257-270: 265-266). Trotzdem gab es noch immer wohlhabende Männer, die der obersten Censusklasse angehörten 
akkumulation von Frauen und stattdessen die Förderung der Vermögensakkumulation von Männern, die Erhöhung des tributum zur Sanierung des Militärhaushalts als Reaktion auf den Einfluss der sine manu-Ehe, als Anreiz zur tutela legitima, als Beschneidung des Instituts der Einsetzung von extranei als Erben, zum Schutz vor der Aufsplittung großer Vermögen, zur Fortführung der sacra familiaria, zur Einschränkung von Luxus, als antifeministische und antiemanzipatorische Repression. ${ }^{31}$

Es kann m. E. vermutet werden, dass die Senatorenschaft unterschiedliche Motive mit der lex Voconia verfolgte. Einigkeit wird darüber bestanden haben, dass sie dieses Gesetz als probate Maßnahme erachteten, um drängende soziale und ökonomische Probleme des 3. und 2. Jahrhunderts v. Chr. anzugehen. Dabei dürfen nicht pauschal misogyne Motive angenommen werden. Denn auch in den Jahren nach dem Erlassen der lex Voconia wurden ihre Schlupflöcher und Schleichwege nicht beseitigt, obwohl sie spätestens jetzt nach einigen Jahren Praxis - bekannt waren. M.E. wurde mit der lex Voconia die Steigerung des Sozialprestiges der biologischen Familie indirekt kritisiert, indem man der Frau - Ehefrau oder Tochter - superbia und luxuria vorwarf. Im Zuge der höheren Mitgiften wurde auch die Ehe sine manu populärer, denn auf diesem Weg erschien die Tochter weiter unter den sui heredes ihres Vaters und die biologische Familie verlor ihr Vermögen nicht an die Familie des Ehemannes. ${ }^{32}$

und ihre militärischen Pflichten erfüllten: „This, I believe, was the principal motive for the passage of the lex Voconia," so Sarah Pomeroy (1976, a.a. O. (Anm. 6), 222).

31 Vgl. u.a. Van der Meer 1996, a.a.O. (Anm. 6), 53-71; Weishaupt 1999, a.a. O. (Anm. 6), 129141; Vigneron und Gerkens 200o, a. a. O. (Anm. 6), 107-121; Vigneron 1983, a.a. O. (Anm. 6), 140-153; Pölönen 1999, a.a.O. (Anm. 6), 111-131; Dixon 1985, a.a.O. (Anm. 6), 519-534; Pomeroy 1976, a. a. O. (Anm. 6), 221-222; Evans 1991, a. a. O. (Anm. 6), 75ff.; Hähnchen 2012, a. a. O. (Anm. 6), 44-52; Weishaupt 1999, a. a. O. (Anm. 6), 128-141; U. Manthe, ,Das Erbrecht der römischen Frauen nach der lex Papia Poppaea und die ratio Voconiana', in: P. Nève und C. Coppens (Hrsg.), Vorträge gehalten auf dem 28. Deutschen Rechtshistorikertag Nimwegen (Nimwegen 1992), 33-47: 40-42; Baltrusch 1989, a. a. O. (Anm. 6), 75-77; Sirks 1994, a. a. O. (Anm. 6), 183-186; McClintock 2013, a.a. O. (Anm. 6), 188 f.

32 Vgl. Evans 1991, a.a. O. (Anm. 6), 8o; Pölönen 1999, a.a. O. (Anm. 6), 127. Zur Zeit der lex Voconia war die manus-Ehe noch die Regel, doch bereits für Gaius ist die manus-Ehe zu Beginn des Prinzipats ein historisches Relikt (Gai. inst. 1,109-113; vgl. Van der Meer 1996, a. a. O. (Anm. 6), 51 sowie Anm. 58; Pomeroy 1976, a.a. O. (Anm. 6), 222; Evans 1991, a.a.O. (Anm. 6), 78-79). Coen van Galen (2016, a. a. O. (Anm. 1), 28-31, 171-180) verortet den Paradigmenwechsel hinsichtlich der Formen der Eheschließung um die des 1. Jahrhunderts v. Chr. 


\section{Die reiche, geschminkte und gut gekleidete Frau mit Schmuck} als Chiffre für Wandel und Gefahr?

\subsection{Zwischen domus und levitas - Die Insignien einer römischen Frau}

Die Tugenden einer römischen Frau waren auf die domus als traditionellem und idealisiertem Wirkungsbereich begrenzt, wie uns u. a. inschriftliche Belege zutragen: „[...] lanifica pia pudica frugi casta domiseda “. ${ }^{33}$ Die Stein gewordenen laudationes funebris dienten einerseits dem self-fashioning des Ehemannes. ${ }^{34}$ Andererseits konnten sowohl Werner Riess als auch Emily Hemelrijk und andere aus zahlreichen Inschriften einen Kanon an weiblichen Tugenden extrahieren, wobei in den meisten Fällen castitas, pudicitia, modestia, obsequium, lanificium und decus muliebris genannt wurden. ${ }^{35}$ Diesen Tugenden wird eine eklatante Schwäche der römischen Frau gegenübergestellt. Ausgangspunkt dieser levitas- und fragilitas-Konzeption ist ihre geringere körperliche Kraft, dem zwei weitere Aspekte jenseits der Physis angefügt werden: Eine römische Frau benötigte einerseits männliche Hilfe zum Ausgleich geistiger Insuffizienz und andererseits männliche Disziplinierung zur Kontrolle charakterlicher Schwäche. Diese naturgegebene Schwäche einer Frau (fragilitas) würde nach Justinian ihre ratio verlangsamen und außerdem in einem wankelmütigen Gemüt (levitas animi) zum Ausdruck kommen. ${ }^{36}$ Die Transformation

33 CIL 6.11602 = ILS 84O2: „[...] spann Wolle, [war] fromm, züchtig, ordentlich, rein und häuslich."

34 Vgl. CIL 6.1527; 37053; AE 1951.2; W. Riess, ,Rari exempli femina: female virtutes on roman funerary inscriptions', in: S.L. James und S. Dillon (Hrsg.), A Companion to Women in the Ancient World (Malden, Oxford, Chichester 2012), 491-501: 495-497; D. Flach, Die sogenannte Laudatio Turiae. Einleitung, Text, Übersetzung und Kommentar (Darmstadt 1991), $2 \mathrm{ff.}, 12,15$; E.A. Hemelrijk, ,Masculinity and femininity in the Laudatio Turiae', Classical Qarterly 54 (2004), 185-197; J. Osgood, Turia. A Roman Woman's Civil War (Oxford 2014); H. Lindsay, ,The man in Turia's life, with a consideration of inheritance issues, infertility, and virtues in marriages in the ist c. B.C., Journal of Roman Archaeology 22 (2009), 183-198; 185-189; W. Kierdorf, Laudatio funebris. Interpretationen und Untersuchungen zur Entwicklung der römischen Leichenrede, Beiträge zur klassischen Philologie 106 (Meisenheim am Glan 1980), 33-48.

35 Vgl. Riess 2012, a. a. O. (Anm. 34), 491-5o1; Hemelrijk 2004, a. a.O. (Anm. 34), 188; B. von Hesberg-Tonn, Coniunx carissim. Untersuchungen zum Normcharakter im Erscheinungsbild der römischen Frau (Stuttgart 1983), 103-104; Lindsay 2009, a. a. O. (Anm. 34), 183-198; H. Lindsay, ,The Laudatio Murdiae: its content and significance', Latomus 63 (2004), 8897; CIL 6.1527; Liv. 1.57.6-11, 1.58-6o. Werden einer Frau jedoch männliche Tugenden im Übermaß attestiert, dann ist auch das nicht zuträglich für ihre Reputation (vgl. Hemelrijk 2004, a.a. O. (Anm. 34), 191-196).

36 Vgl. Cod. Iust. 4.29.22. Zur Anfälligkeit von Frauen für Krankheiten vgl. Dig. 27.10.9, 22.6.9pr.; 48.16.1.10, 49.14.18pr.; Cod. Theod. 12.1.137.1, 9.14.3.2. 
des levitas- und fragilitas-Konzepts von einem literarischen Topos hin zu einer allgemein akzeptierten Aussage zeichnete Suzanne Dixon bereits in einer Studie von 1984 sorgfältig nach. ${ }^{37}$ Die levitas der Frauen wird jedoch nicht nur als Wankelmütigkeit oder Schwäche des Geistes gesehen, sondern sehr wohl auch als Unfähigkeit, die eigenen körperlichen Bedürfnisse zu kontrollieren, woraus ein allgemeiner Schutz für Frauen abgeleitet wird. Dieser Ansatz ermöglicht es, Frauen im öffentlichen Raum von politischen Funktionen und staatlichen Ämtern auszuschließen und sie für private Rechtsgeschäfte unter die auctoritas eines Tutors (tutela mulierum) zu stellen. ${ }^{38}$ Männer hingegen seien von Natur aus beherrschter und rationaler, was auch ihr Handeln in allen Lebensbereichen bestimmen würde. Gleichzeitig prädestiniere sie diese Charaktereigenschaft auch zur Kontrolle über Frauen, was zur „Konstruktion männlicher Selbstverständlichkeit“ führte. ${ }^{39}$ In dieser Ideenwelt gingen Psychologie und Physiologie eine folgenreiche Allianz ein und erzeugten so ein Bild, dass ein starker Geist nur in einem starken Körper wohnen könnte, wobei beides durch den Mann verkörpert würde. Gleichzeitig erwuchsen weibliche Zerbrechlichkeit und Schwäche, kombiniert mit ihrer Unbeständigkeit und Wankelmütigkeit, zu einem pathologischen und bedrohlichen Zustand.

Auch Historiographen, Literaten und Juristen bedienten sich dieser Stereotype und Rollenbilder und transferierten diese in andere literarische Gattun-

37 Vgl. S. Dixon, Infirmitas sexus: womenly weakness in Roman law', Tijschrift voor Rechtsgeschiedenis $5^{2}$ (1984), 343-371.

38 Vgl. Dig. 50.17.2pr.; Cic. Mur. 27: Mulieres omnes propter infirmitatem consilii maiores in tutorum potestate esse voluerunt [...] - Unsere Vorfahren wünschten, daß alle Frauen wegen der Unsicherheit ihres Urteils der Gewalt eines Vormundes unterstünden [...] (M. Tullius Cicero, Sämtliche Reden II, eingel., übers. und erläut. von M. Fuhrmann, Bibliothek der Alten Welt (Zürich, Stuttgart 1970)). Mit dieser Aussage gibt Cicero nicht an, ob Frauen an einem Defizit leiden, das eine tutela mulierum notwendig machen würde. Benke (2002, S. 476) stützt sich auf Gaius (Inst. 1.189-19o) und geht davon aus, dass seit der späten Republik die tutela mulierum weniger strikt praktiziert wurde (vgl. ebs. B. Feldner, ,Zur Vermögensverwaltung durch Frauen im klassischen römischen Recht', in S. Meder, A. Duncker und A. Czelk (Hrsg.), Frauenrecht und Rechtsgeschichte. Die Rechtskämpfe der deutschen Frauenbewegung (Köln, Weimar, Wien 2006), 1-20: 4; Levick 2012, a.a.O. (Anm. 1), 99-100; Gardner 1995, a. a. O. (Anm. 1), 377). Seit der späten Republik und in der frühen Kaiserzeit erlangten Frauen mehr Selbständigkeit in geschäftlichen Angelegenheiten, da die tutela mulierum meist nur noch als bloße Formsache praktiziert wurde. Zum Ausschluss von Frauen von der Bekleidung öffentlicher Ämter vgl. Dig. 50.17.2pr.-1; Benke 2012, a. a. O. (Anm. 1), 220; Benke und Halbwachs 2002, a. a. O. (Anm. 1), 472; Gardner 1995, a.a. O. (Anm. 1), 377-378; Levick 2012, a. a. O. (Anm. 1), 98; Van Galen 2016, a.a. O. (Anm. 1), 51.

Vgl. Höbenreich und Rizzelli 2003, a. a. O. (Anm. 18), 40. 
gen. ${ }^{40}$ Zur Verdeutlichung dieses stereotypisierten Rollenverhaltens und der als Bedrohung empfundenen unterstellten Rolleninversion wurden Attribute und Eigenschaften eingebracht, die die schwache Frau beschreiben sollten. In der Selektion bestimmter Momentaufnahmen aus dem weiblichen Alltag erfahren diese Ausschnitte eine Überhöhung und Verzerrung. Eines dieser Bilder ist das der sehr wohlhabenden Frau, die einerseits ihren Reichtum - wahrscheinlich großen Landbesitz und Erbschaften - durch zahlreiche Schmuckstücke und kostbare Kleidung nach außen trägt. ${ }^{41}$ Andererseits werden diese Dinge auch als Symbol der weiblichen Rolle in der Gesellschaft gelesen. ${ }^{42}$

Im Kontakt mit dem sozialen Umfeld, mit den Anderen, ist der Körper das zuerst Sichtbare und deshalb wichtig für die soziale Identität und ihre Außenwirkung. In der männlichen Narration zu Frauen liegt der Fokus u.a. auf Schmuck, Kleidung und Körperpflege sowie auf damit in Zusammenhang stehenden Utensilien. ${ }^{43}$ Das stereotypisierte Bild der römischen Frau entstand in einem Spiegel, war konstruiert als Objekt männlicher Interessen und Motive: „Through descriptions of women's bodily adornment, a constellation of negative values is attached to the female sex. ${ }^{\text {"4 }}$ Die Fokussierung auf die Frau in der männlichen Narration lässt sie als auf Äußerlichkeiten und Körperlichkeiten reduziertes Individuum erscheinen. ${ }^{45}$ Auch ist auffällig, dass Frauen lediglich über Äußerlichkeiten definiert wurden: Einerseits wird der Frau vorgeworfen, dass sie Aufwand betreibe, um schön und attraktiv zu wirken, andererseits erscheint die geschminkte und Schmuck tragende Frau als probate Chiffre, um ihren hohen sozialen Stand zu indizieren. ${ }^{46}$ Des Weiteren wird sie als Gefahr

$40 \quad$ Gardner 1995, a.a. O. (Anm. 1), 378.

41 Vgl. McClintock 2013, a.a.O. (Anm. 6), 183.

42 Vgl. McClintock 2013, a.a. O. (Anm. 6), 18-185; Polyb. 31.26.1; Diod. 31.27.3-4.

43 Vgl. Gardner 1995, a. a. O. (Anm. 1), 381-382. Der Aspekt der Körperpflege erhält in Zusammenhang mit Männern eine andere Konnotation: Die Körperpflege, cultus, sei notwendig für jeden römischen Bürger, da sich der Mann in der Öffentlichkeit aufhielt und Ämter bekleidete. Durch den männlichen Körper und sein Erscheinungsbild wurde also Menschsein und Bürgersein ausgedrückt (vgl. M. Wyke, ,Woman in the mirror: the rhetoric of adornment in the Roman world', in: L.J. Archer, S. Fischler und M. Wyke (Hrsg.), Women in Ancient Societies. An Illusion of the Night (London 1994), 134-151: 135; Liv. 2.23.4). Zu einem gepflegten Äußeren gehörten u.a. gewaschenes und geschnittenes Haar und Bart. Zur angemessenen Kleidung im Sinn des mos maiorum vgl. Gell. 11.2 und bei der Amtsbewerbung vgl. u. a. Liv. 36.22-27, 37.57.9-15.

44 Vgl. Wyke 1994, a.a. O. (Anm. 43), 136.

45 Vgl. Plaut. Poen. 203-231; Iuv. 6.461-674.

46 Vgl. Wyke 1994, a. a.O. (Anm. 43), 137; Hemelrijk 2004, a.a.O. (Anm. 34), 188; H. Matthäus, ,Untersuchungen zu Geräte- und Werkzeugformen aus der Umgebung von Pompeji‘, Berichte der Römisch-Germanischen Kommissionen 65 (1984), 73-158: 92; L. Shumka, ,Designing women: the representation of women's toiletries on funerary monuments in Roman 
für die res publica erachtet: „[...] men are given the power to paint gender definitions on to the bodies of women and then to erase the potential danger which those definitions articulate. ${ }^{47}$

\subsection{Regulierungsversuche: Die lex Voconia in der Tradition der lex Oppia?}

Wenn sich hinsichtlich des Erbeinsetzungsverbots von Frauen vermögensrelevante Folgen ausschließen lassen, dann kann vermutet werden, dass Frauen von der Rolle als testamentarische Erbinnen ausgeschlossen werden sollten aufgrund der Wirkkraft ihrer Rolle in Privatheit und Öffentlichkeit. ${ }^{48}$ Welche Rollenbilder und welches Genderverständnis lagen diesen Überlegungen, die zur lex Voconia führten, zugrunde? Idealiter war die römische Frau der Oberschicht limitiert auf bestimmte Rollen und Relationen, auf ein spezifisches Rollenverhalten und auf ausgewählte Tugenden, was in einer Überhöhung ihres Wirkungsbereichs domus ersichtlich wird. In Anlehnung an diese Topoi und in Abgrenzung durch die Chiffre der geschminkten und Schmuck tragenden Frau sollte das Sozialprestige und die Reputation des Erben exklusiv Männern vorbehalten bleiben. ${ }^{49}$

Diese Argumentation möchte ich zunächst an einem anderen Beispiel verdeutlichen: Eine vergleichbare Motivation kann nämlich auch der lex Oppia aus dem Jahr 215 v. Chr. zugeschrieben werden, gegen die sich 195 v. Chr. der Protest römischer Frauen richtete. ${ }^{50}$ Im Kontext des 2. Punischen Kriegs und der

Italy‘, in:J. Edmondson und A. Keith (Hrsg.), Roman Dress and the Fabrics of Roman Culture (Toronto 2008), 172-191; Sen. Cons. 16.

47 Wyke 1994, a.a.O. (Anm. 43), 146; vgl. Levick 2012, a.a.O. (Anm. 1), 102. Die Keuschheit und Reinheit einer Frau genauso wie ihre sexuelle Unantastbarkeit symbolisierten die Unverletzbarkeit Roms. „The honorable female body stood for the Roman body politic as a whole. [...] pietas [was] regarded as the pivotal core of Rome's social and political cohesiveness" (Riess 2012, a. a. O. (Anm. 34), 492).

48 Nikolaus Benke (2002, 505) greift hier eine Argumentation von Edouard Lambert (La tradition romaine sur la succession des formes du testament devantl'histoire comparative (Paris 1901), 100-103) auf, doch mit einer anderen Erklärung: Die lex Voconia sollte ein angeblich altrömisches Prinzip wiederherstellen, demzufolge Frauen überhaupt nicht als Erbinnen eingesetzt werden durften (vgl. ebs. Vigneron 1983, a.a. O. (Anm. 6), 143-144; Vigneron und Gerkens 2000, a. a. O. (Anm. 6), 107-121; Gai. Inst. 2.274; Dig. 16.1.1.1; 50.16.195.2, 50.16.195.45; im Gegensatz dazu vgl. Weishaupt 1999, a.a.O. (Anm. 6), 128-141; Manthe 1992, a.a. O. (Anm. 31), 40-42).

49 Vgl. Benke 2002, a. a. O. (Anm. 6), 506.

$50 \quad$ Vgl. Liv. 34.2.8-12, 34.6.11-16. Die lex Oppia in Livius Darstellung unterrichtet uns nicht etwa über die römische Republik des 3. und 2. Jahrhunderts v. Chr., sondern vielmehr über die Zeit des Livius (vgl. B. Hopwood, ,Livy and the Repeal of the lex Oppia', in P. Keegan (Hrsg.), Text, Artifact Context. The Interactions of Literature, Material Culture and Mentality 
Gefährdung Roms war mit der lex Oppia ein Plebiszit erlassen worden, das allen Römerinnen untersagte, einen luxuriösen Lebensstil im öffentlichen Raum zu zeigen, was sich im Falle von Frauen - so zumindest das Verständnis römischer Männer - u.a. an einem übermäßigen Gebrauch von Schmuck und auffälliger Kleidung zeigte. ${ }^{51}$ Während der Kriege Roms im 3. und 2. Jahrhundert v. Chr. und der damit verbundenen Absenz der Männer übernahmen Frauen - Mütter, Ehefrauen und Töchter - vermehrt Aufgaben, die ursprünglich der männlichen Domäne zugerechnet worden waren. ${ }^{52}$ Diese scheinbare Inversion von althergebrachten Asymmetrien, die für konservative Männer wie Cato saturnalischen Verhältnissen gleichkam, führte zu konservativen und konservierenden Reaktionen, wie sie in der lex Oppia und lex Voconia gesehen werden können. ${ }^{53}$

Dem Protest der Frauen Roms gegen die lex Oppia stellt Livius in der Figur des Cato einen Verteidiger des Gesetzes mit einem dystopischen Angstszenario entgegen: Frauen müssten repressiv behandelt werden, um die Gefahr zu bannen, dass sie noch einflussreicher werden und noch auffälliger im öffentlichen Raum erscheinen würden. ${ }^{54}$ In seiner Replik auf Catos Rede betont Valerius, dass Frauen auch nach der Abschaffung der lex Oppia keinerlei Gefahr darstellen würden: Ihr Interessenshorizont würde sich auf ihr Aussehen, ihren

in the Ancient World (Armidale 20o1), 121-139: 122; Baltrusch 1989, a. a. O. (Anm. 6), 56 f.; Van Galen 2016, a. a. O. (Anm. 1), 22 ff.; 171; E.A. Hemelrijk, ,Women's demonstrations in Republican Rome', in J. Blok and P. Mason (Hrsg.), Sexual Asymmetry. Studies in Ancient Society (Amsterdam 1987), 217-240). Zur lex Iulia Caesaris und lex Iulia de maritandis ordinibus vgl. Baltrusch 1989, a. a. O. (Anm. 6), 59-6o. Zu einer weiteren überlieferten Demonstration römischer Bürgerinnen vgl. App. civ. 4,32-34; Van Galen 2016, a. a. O. (Anm. 1), 20-24. Vgl. Liv. 34.7.1-3. Liv.34.1.2-3. Die zahlreichen Feldzüge forderten einen hohen finanziellen Einsatz. In dieser Zeit wurden weitere Luxusgesetze erlassen, die den verschwenderischen Lebensstil einzelner anprangerten und gleichzeitig die Entbehrungen der Gemeinschaft vor Augen führten.

$5^{2}$ Vgl. Baltrusch 1989, a. a. O. (Anm. 6), 54-58.

53 Gellius (17.6.1, 17.8-10) schildert den Fall einer manus-freien Ehe, über den sich Cato sehr echauffiert haben soll: In diese Ehe brachte die Frau eine Mitgift ein, aber nicht ihr weiteres Vermögen, das wahrscheinlich von ihrer biologischen Familie stammte. Sie gewährte ihrem Mann ein Darlehen, das dieser aber zurückzahlen sollte. Cato störte sich an dem Verhalten der Frau, ihrem Ehemann nicht das gesamte Vermögen zur Verwaltung überlassen zu haben, sowie an ihrer Forderung nach Zurückzahlung des Darlehens (vgl. Höbenreich und Rizzelli 2003, a. a. O. (Anm. 18), 108-110; G. van Niekerk, ,Stereotyping women in Ancient Roman and African societies: a dissililarity in culture', Revue Internationale des droits de l'antiquité 47 (2000), 365-379: 371-374).

54 Vgl. Liv. 34.2.13-14, 34.3-2; Benke 2002, a.a.O. (Anm. 6), 509; McClintock 2013, a.a.O. (Anm. 6), 185-186; Höbenreich und Rizzelli 2003, a.a. O. (Anm. 18), 99 ff.; Vigneron und Gerkens 200o, a.a.O. (Anm. 6), 107-121; Wyke 1994, a.a.O. (Anm. 43), 139-140; Hähnchen 2012, a.a.O. (Anm. 6), 45; Levick 2012, a.a.O. (Anm. 1), 102; Hopwood 2001, a.a.O. (Anm. 50), 124-128; Van der Meer 1996, a.a. O. (Anm. 6), 47. 
Schmuck und ihre Kleidung beschränken, denn das seien ihre Insignien. ${ }^{55}$ Beide berufen sich, obwohl sie unterschiedliche Positionen einnehmen, auf althergebrachte Argumentationsstränge und zeichnen in ihren Plädoyers das Bild der tugendhaften römischen Frau, die der domus verhaftet bleiben sollte, als Gegenentwurf: Ihre Chiffre ist die Wolle spinnende Frau. ${ }^{56}$ Damit stellt Livius in seiner Narration die Ordnung der römischen Gesellschaft wieder her.

Das Konzept der römischen Familie gründete sich vor allem auf die patria potestas, mit dem pater familias als ausführendem Organ. Es existierte keine vergleichbare Macht und Rolle für eine Frau innerhalb der familia und damit waren römische Frauen ausgeschlossen, andere zu repräsentieren. ${ }^{57}$ Der Ausschluss von Frauen von der Repräsentation wirkte daher konservierend, d.h. alte, überkommene Strukturen wurden auf diese Weise manifestiert bzw. zementiert. Ihre visuelle Expression zeigt sich in der geschminkten und Schmuck tragenden Frau; mit der lex Oppia und der lex Voconia sollte sie - als Chiffre eines Wandels und als role model einer neuen Zeit - aus dem öffentlichen Raum entfernt werden. Wurde eine Frau Erbin, dann war es ihre Aufgabe, den letzten Willen des Erblassers zu realisieren: In diesem Zusammenhang war sie Gläubigerin ererbter Forderungen, Schuldnerin übernommener Verbindlichkeiten und zuständig für die Verteilung von Legaten und Fideikommissen. ${ }^{58}$ In der Folge würde sie als selbständige und Entscheidungen treffende Frau im öffentlichen Raum in Erscheinung treten, in der Privatheit könnte sie auf diesem Weg ihre Position innerhalb der familiären Hierarchie stärken. Mit dieser vermeintlichen Inversion weiblichen Rollenverhaltens würde die Ursituation funktionierender gesellschaftlicher Kohäsion infrage gestellt und nur über patriarchalische Strukturen sei diese zu erhalten. ${ }^{59}$

55 Vgl. Liv. 34.7.5-13. Zu den Reden des Cato und des Valerius vgl. Hopwood 2001, a.a.O. (Anm. 50), 122-139; Höbenreich und Rizzelli 2003, a.a. O. (Anm. 18), 102 ff.; Wyke 1994, a. a. O. (Anm. 43), 139-140; H. Cancik-Lindemaier, ,Die Welt der Frau', Metis 14 (1998), 2129. McClintock 2013, a.a.O. (Anm. 6), 186: „The repeal of the lex Oppia resulted in the restitution of their jewels. The restored insignia were the emblem of a social compromise between the sexes."

56 Vgl. Suet. Aug. 64.2, 73 .

57 Vgl. Benke 2012, a. a. O. (Anm. 1), 224; Dixon 1995, S. 377; 384-388.

58 Vgl. McClintock 2013, a. a. O. (Anm. 6), 192: „Evidently as heirs they had gained independence in managing the key-estates of Rome.“

59 Coen van Galen (2016, a. a. O. (Anm. 1), 114) macht in seiner Studie „Women and Citizenship in the Late Roman Republic and the Early Empire“ die Unsichtbarkeit der römischen Bürgerin sichtbar: „In societies with a focus on male inheritance line women are also somewhat suspect." 
Nach Jack Goody bestimmten die in einer Gesellschaft vorherrschenden Formen der Vererbung auch den Umgang der Gesellschaft mit Frauen: Einerseits waren sie potentielle Erbinnen, andererseits wurde ihnen diese Rolle nicht immer vollumfänglich zugestanden, ihr Verhalten in Öffentlichkeit und Privatheit reglementiert. ${ }^{61}$ Eine der Klauseln der lex Voconia schließt Frauen als Erbinnen aus den letzten Willen der Erblasser der 1. Censusklasse wegen der mit Familiendominanz, Repräsentation in der Öffentlichkeit, politischem Potential und Sozialprestige verbundenen Rolle der testamentarisch bestimmten Erben aus. Der lex Voconia liegt m. E. ein idealisiertes und stereotypisiertes Rollenbild und Gender-Verständnis zugrunde, dass römische Frauen der Oberschicht auf ein spezifisches Rollenverhalten und bestimmte Tugenden limitierte, was in einer Überhöhung ihres Wirkungsbereichs domus ersichtlich wird. In Anlehnung an die Topoi der tugendhaften Frau und in Abgrenzung durch die Chiffre der geschminkten und Schmuck tragenden Frau sollte auch das Sozialprestige und die Reputation des Erben exklusiv Männern vorbehalten bleiben. Wie kann iustitia in diesem Kontext verortet werden?

Gerechtigkeit wird angesichts von Konflikten, Umbrüchen und Verknappungen herausgefordert. Die Expansion Roms und die Feldzüge bewirkten auch einen Wandel im Inneren, im Sozialen, wobei Veränderungen und die damit einhergehende Aktivität und Neuausrichtung der sozialen Rolle Männern zugestanden wurde, Frauen jedoch nicht. Während die Rolle des Mannes und sein Handeln weiterhin mit dem mos maiorum im Einklang standen, galt das nicht für Frauen. Durch ihr in der Literatur gezeichnetes Bild erhält der Transformationsprozess ein Gesicht, nämlich das der geschminkten und Schmuck tragenden Frau, die ein verändertes Sozialprestige verkörpert. Für konservative Kreise - wie Cato und Valerius in Livius Ab urbe condita - gilt sie als Chiffre einer dystopischen Gegenwart und gleichzeitig als Prophezeiung einer ebensolchen Zukunft. Um dies zu verdeutlichen wurden traditionelle Stereotype bemüht: Das Rollenverhalten der körperlich und geistig schwachen Frau wurde kombiniert mit der aktuellen Situation. In diesem Dialog wurde ein negatives und warnendes Rollenbild kreiert, also die geschminkte und

6o Dig. 50.16.195.5: „Eine Frau ist aber sowohl Oberhaupt und Anfang als auch das Ende ihrer eigenen Familie." Vgl. Benke 2002, a.a. O. (Anm. 6), 507.

61 Vgl. J. Goody, ,Inheritance, property and marriage in Africa and Eurasia', Sociology 3 (1969), 55-76; J. Goody, The Oriental, the Ancient and the Primitive: Systems of Marriage and the Family in the Pre-industrial Societies of Eurasia (Cambridge 199o), 70-71, 78; Van Galen 2016, a. a. O. (Anm. 1), $43 \mathrm{ff}$. 
Schmuck tragende Frau, deren Äußeres Reichtum und ein Mehr an Unabhängigkeit zum Ausdruck bringt. Sie steht als Chiffre für eine Veränderung, die ein Teil der Senatorenschaft nicht wollte und nicht goutierte. Das Verhalten, das ihrer Rolle zugeschrieben wird, ist Fiktion und dient der Abgrenzung.

Die Auseinandersetzung um die Rolle und das Rollenverhalten von römischen Frauen wird mit Rollenbildern, Stereotypen und Chiffren geführt, wobei sich die Frage stellt, ob die lex Voconia ungerecht war. Gerechtigkeit kann als Prinzip einer ausgleichenden Ordnung in der Gesellschaft gesehen werden, als moralisch-ethische Kohäsion von Recht und Gesetz. Findet der Ausgleich nicht statt, wird die soziale Kohäsion aufgehoben, dann werden Prozesse und Entscheidungen als ungerecht wahrgenommen. In seiner staatstheoretischen Schrift De re publica bezeichnet Cicero die lex Voconia als „voller Unrecht gegen die Frauen", als ein Gesetz, das nur Männern nützen würde. ${ }^{62}$ Auch in seiner zweiten Rede gegen Verres, die nicht mehr vor Gericht vorgetragen, sondern lediglich in schriftlicher Form publiziert wurde, erachtete Cicero das Erbeinsetzungsverbot als ungerecht, wobei diese Einschätzung einerseits aus der Intention der Gerichtsrede erwächst, nämlich die Deklassierung des Verres durch Habgier und seine Verurteilung. ${ }^{63}$ Andererseits wird wohl auch Ciceros persönliche Situation eine Rolle gespielt haben bzw. er nutzte sie, um seine Argumentation zu stärken und überzeugend zu präsentieren. Denn ähnlich wie der Fall des Erblassers Publius Annius Asellus, der seine Tochter Annia als sein einziges Kind zur Universalerbin machte sowie einen Verwandten namens L. Annius als Ersatzerben einsetzte, hatte Cicero im Jahre 70 v. Chr. nur seine Tochter Tullia. ${ }^{64}$

Gab es also eine genderspezifische iustitia im römischen Erbrecht? Jede Gerechtigkeit hat unterschiedliche Gestaltungsmöglichkeiten und nimmt deshalb eine unterschiedliche Art von Bewertung vor: sozial (gut für irgendetwas), normativ (gut für jemanden) und moralisch (gut für jeden einzelnen). Den gesamten Bereich der Moral deckt Gerechtigkeit aber nicht ab. ${ }^{65}$ Sowohl Platon und Aristoteles als auch Ulpian sehen in Gerechtigkeit einerseits eine alles umfassende Tugend, andererseits aber auch ein Regularium für interpersonale Beziehungen. Konservative Kreise erachteten den mos maiorum als Richtli-

62 Vgl. Cic. Rep. 3.10.17; Weishaupt 1999, a.a. O. (Anm. 6), 18-19; Gardner 1986, a.a. O. (Anm. 4), 175; Hähnchen 2012, a.a. O. (Anm. 6), 46. Auch Augustinus von Hippo (Civ. 3.21) äußert ähnliche Vorbehalte gegenüber der lex Voconia, wobei er sich wahrscheinlich auf Ciceros Ausführungen in De re publica stützte.

63 Vgl. Cic. Verr. 2.1.43.104-114.

64 Vgl. Van der Meer 1996, a.a.O. (Anm. 6), 73-79; Weishaupt 1999, a.a.O. (Anm. 6), 8-14; Vigneron 1983, a.a.O. (Anm. 6), 144. Zu weiteren Erwähnungen der lex Voconia bei Cicero vgl. fin. 2.17.55; Balb. 21; Cato 5.14; Cluent. 7.21; Caec. 4.11.

65 Vgl. Höffe 2001, a.a. O. (Anm. 13), 3о. 
nie für gerechtes Handeln. Da die neue Selbständigkeit und Sichtbarkeit der römischen Frau als nicht deckungsgleich mit den altehrwürdigen mores deklariert wurde, war nicht das Vorgehen gegen Frauen ungerecht, sondern vielmehr deren Verhalten. Cicero spricht zwar von der Ungerechtigkeit der lex Voconia, doch erlebt der Leser die ciceronische Empfindung entweder als Bestandteil einer juristischen Argumentation zulasten des Verres oder als Resultat seiner persönlichen Situation. Er lässt jedoch keine auf Prinzipien der Gleichheit und Gleichberechtigung gegründete Ablehnung des voconischen Gesetzes erkennen. Gleichzeitig fand die Frage nach Gerechtigkeit in Zusammenhang mit der lex Voconia in einem spannungsgeladenen Gefüge von Ideal und Realität statt: Das dem voconischen Erbeinsetzungsverbot von Frauen zugrundeliegenden

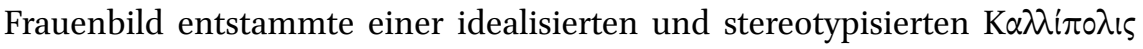
und fungierte gleichzeitig als Richtlinie für soziales Interagieren: Gerechtigkeit wird darin ersichtlich, dass jede und jeder die ihr oder ihm zugewiesenen Aufgaben erfüllt. 